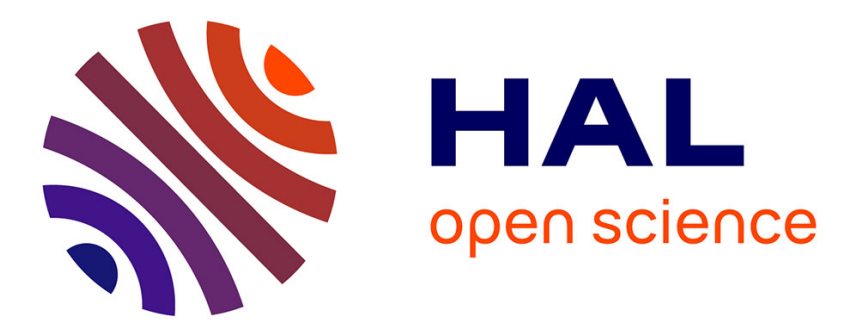

\title{
Dispersion of charged tracers in charged porous media
}

Benjamin Rotenberg, I. Pagonabarraga, Daan Frenkel

\section{To cite this version:}

Benjamin Rotenberg, I. Pagonabarraga, Daan Frenkel. Dispersion of charged tracers in charged porous media. EPL - Europhysics Letters, 2008, 83, pp.34004. 10.1209/0295-5075/83/34004 . hal-00369645

\section{HAL Id: hal-00369645 \\ https://hal.science/hal-00369645}

Submitted on 20 Nov 2018

HAL is a multi-disciplinary open access archive for the deposit and dissemination of scientific research documents, whether they are published or not. The documents may come from teaching and research institutions in France or abroad, or from public or private research centers.
L'archive ouverte pluridisciplinaire HAL, est destinée au dépôt et à la diffusion de documents scientifiques de niveau recherche, publiés ou non, émanant des établissements d'enseignement et de recherche français ou étrangers, des laboratoires publics ou privés. 


\title{
Dispersion of charged tracers in charged porous media
}

\author{
B. Rotenberg ${ }^{1,2}$, I. Pagonabarraga ${ }^{3}$ and D. Frenkel ${ }^{1,4}$ \\ 1 FOM Institute for Atomic and Molecular Physics - Kruislaan 407, 1098 SJ Amsterdam, The Netherlands \\ 2 ANDRA - Parc de la Croix Blanche, 1/7 rue Jean Monnet 92298 Châtenay Malabry cedex, France \\ 3 Departament de Fisica Fonamental, Universitat de Barcelona - Carrer Martí i Franqués 1, 08028-Barcelona, Spain \\ 4 Department of Chemistry, University of Cambridge - Lensfield Road, Cambridge CB2 1EW, United Kingdom
}

PACS 47.1.-j - Computational methods in fluid dynamics

PACS 47.56. $+r-$ Flow through porous media

PACS $47.57 . j \mathrm{~b}$ - Electrokinetic effects

\begin{abstract}
We report a lattice-Boltzmann scheme to compute the dispersion of charged tracers in charged porous media under the combined effect of advection, diffusion and electro-migration. To this end we extend the moment propagation approach, introduced to study the dispersion of neutral tracers [Lowe et al., Phys. Rev. Lett. 77, 4552 (1996)], to include the effect of electrostatic forces. This method allows us to compute the velocity autocorrelation function of the charged tracers with high accuracy. The algorithm is validated studying the dispersion coefficient in the case of electro-osmotic flow in a slit without added salt. We find excellent agreement between the numerical and analytical results. This method also provides the full time dependence of the diffusion coefficient, including for charged tracers. We illustrate on the slit case how $D(t)$, which is measured by NMR to probe the geometry of porous media, reflects how the porosity explored by tracers depends on their charge.
\end{abstract}

The transport of charged particles in charged porous media, under the combined effect of advection, diffusion and migration, is encountered in many situations of technological interest, such as the separation of species by electro-osmotic flow in capillaries, microfluidic devices and ion-exchange membranes. Contamination of soils by toxic or radioactive waste, which often consist of charged species, are examples of transport of charged tracers in porous media with considerable environmental relevance. Similarly, a better understanding of the electro-kinetic removal of such contaminants is of considerable practical importance. Even in complex biological systems such as the living cell, electro-kinetic transport (often in the nonlinear regime) plays a relevant role. A saturated porous media consists by definition of a solid phase and a fluid phase. At mean-field level and neglecting the finite size of the particles, the dynamics of charged species in the fluid phase can be described in terms of the species density $\rho(\mathbf{r}, t)$, which follows a convection-diffusion equation

$$
\begin{aligned}
& \partial_{t} \rho(\mathbf{r}, t)+\nabla \cdot \mathbf{J}(\mathbf{r}, t)=0 \\
& \mathbf{J}=\rho \mathbf{u}-D \nabla \rho+\rho \beta D[q \mathbf{E}-\nabla V]
\end{aligned}
$$

where $D$ is the particle's molecular diffusion coefficient, $q$ its charge, and $\beta=1 / k_{B} T$. In addition to the fluxes due to advection $(\rho \mathbf{u})$ and diffusion $(-D \nabla \rho)$, two forcing terms contribute to the charged species dynamics. The first includes the effect of particle interactions with the rest of the system, as the gradient of the mean-field potential $V$. This potential is in fact the excess part of the tracer chemical potential. It is not necessarily limited to the electrostatic term $q \psi$ where $\psi$ satisfies the Poisson equation $\Delta \psi=\rho_{e l} / \epsilon_{0} \epsilon_{r}$, with $\rho_{e l}$ the charge density, $\epsilon_{0}$ and $\epsilon_{r}$ the void and fluid relative permittivities, respectively. The second contribution accounts for the effect of an external electric field, which does not necessarily derive from a potential, e.g. for infinite systems.

Although the dispersion of tracers could in principle be investigated by studying the spreading of a tracer pulse, the evaluation of the dispersion coefficient requires solving Eq. (1), e.g. with a finite element method, for a large number of initial conditions, which rapidly becomes computationally intractable for complex porous media. An alternative is to evaluate it for each tracer charge $q$ from the tracer velocity auto-correlation function (VACF) as $[1,2]$ :

$$
D_{\gamma \gamma^{\prime}}=\int_{0}^{\infty} \mathrm{d} t\left\langle\left[v_{\gamma}(0)-\bar{v}_{\gamma}\right]\left[v_{\gamma^{\prime}}(t)-\bar{v}_{\gamma^{\prime}}\right]\right\rangle
$$


where $\bar{v}_{\gamma}$ is the average velocity in the direction $\gamma \in$ $\{x, y, z\}$. The issue of averaging over initial conditions remains, but it can be handled both elegantly and efficiently using the moment propagation method introduced for the dispersion of neutral tracers $[1,3,4]$. The purpose of the present paper is to extend this method to the dispersion of tracers experiencing forces, with the express intent to study charged tracers in charged porous media.

Computing the VACF for a tagged particle requires to keep track of its velocity. However, the convectiondiffusion equation does not contain any information on the velocity of individual particles. Therefore, we will describe the fluid dynamics on the basis of the latticeBoltzmann [5] approach, which follows the evolution of the fluid particle distribution function $f(\mathbf{r}, \mathbf{v}, t)$ on a lattice, and allows to recover the hydrodynamic behavior on long distances. Moreover, the distribution function at each node is related to the transition probabilities of a particle moving in the fluid from one node to a neighboring one. This makes it possible to use the same scheme to analyze the VACF of tracers. Such an approach, referred to as moment propagation $[1,3,4]$, has been pursued earlier for neutral tracers in a neutral fluid, and it has been shown to be an extremely efficient computational tool. Moment propagation allows the propagation of any moment of the particle distribution function: It has e.g. been used to analyze the dynamic structure factor in neutral porous media [6]. It has also been extended for neutral species to the case of anisotropic advection-diffusion problems [7].

In order to use the same approach for charged fluids, we need to generalize the underlying lattice-Boltzmann scheme to account for the dynamics of the dissolved electrolyte. The fact that tracers evolve on average following a convection-diffusion equation has been used earlier [8] to take advantage of moment propagation scheme to simulate eq. (1) including the coupling to the electric field to linear order. The particle density is indeed the zeroth moment in velocity space of the distribution function and falls as such into the scope of the moment propagation scheme. As other lattice-Boltzmann schemes, this original algorithm can lead in the presence of moving boundaries to spurious ionic fluxes across the fluid/solid boundaries. To overcome this difficulty, Capuani et al. [9] introduced an alternative approach, the link-flux method, to reproduce the dynamics of charge densities and recover eq. (1). At the price of treating advection on a different level than diffusion and migration, they proposed an algorithm satisfying the detailed balance condition between neighboring nodes at steady-state, thereby allowing at the same time to rigorously cancel the fluxes into the solid and to deal with larger electrostatic potential gradients.

Either of the two methods described above allows for a proper simulation charged fluid dynamics. Our goal is to exploit the moment propagation method beyond the zeroth order moment (density) in order to compute the VACF of charged tracers. Since moment propagation requires a strict probability conservation, we need to (a) avoid particle leakage into the solid and ensure that the detailed balance condition is satisfied at steady-state, while (b) treating advection, diffusion and migration on the same footing. This latter point is essential for the probabilistic interpretation of the LB scheme and the connection with the VACF, as we describe subsequently.

In a lattice-Boltzmann scheme the system is described by a lattice of spacing $\Delta x$ whose nodes are either in the solid or the fluid phase. The particles can move from one node to the neighbouring ones through a finite set of discrete velocities $\left\{\mathbf{c}_{i}\right\}_{i \in\left[1 . . N_{\max }\right]}$. The probability of being at a given node is $P(\mathbf{r}, t)=\rho(\mathbf{r}, t) / \bar{\rho}$, with $\bar{\rho}$ the mean density. The moment propagation algorithm evolves this probability according to

$$
\begin{gathered}
P(\mathbf{r}, t+\Delta t)=\sum_{i} P\left(\mathbf{r}-\mathbf{c}_{\mathbf{i}} \Delta t, t\right) p_{i}\left(\mathbf{r}-\mathbf{c}_{\mathbf{i}} \Delta t, t\right) \\
+P(\mathbf{r}, t) p_{0}(\mathbf{r}, t)
\end{gathered}
$$

where the sum runs over all directions connecting adjacent nodes, while $p_{i}(\mathbf{r}, t)$ stands for the probability of leaving node $\mathbf{r}$ along the direction $\mathbf{c}_{i}$. The fraction of particles remaining on node $\mathbf{r}$ is $p_{0}(\mathbf{r}, t)=1-\sum_{i} p_{i}(\mathbf{r}, t)$. Central in this description is the expression for $p_{i}$, which we generalize to

$$
\begin{aligned}
p_{i}(\mathbf{r}, t)=p_{i}^{a d v}(\mathbf{r}, t)+ & \lambda w_{i}\left\{\frac{1}{4} \beta q \mathbf{E} \cdot \mathbf{c}_{\mathbf{i}} \Delta t\right. \\
+ & \left.\frac{1}{1+e^{-\beta\left[V(\mathbf{r})-V\left(\mathbf{r}+\mathbf{c}_{\mathbf{i}} \Delta t\right)\right]}}\right\}
\end{aligned}
$$

if $\mathbf{r}+\mathbf{c}_{\mathbf{i}} \Delta t$ belongs to the fluid phase, and 0 otherwise. The weights $w_{i}$ depend on the underlying lattice $[5,10]$.

The first term in Eq. (4) accounts for fluid advection. It is obtained by coupling the tracer motion to a fluid that evolves on the same lattice according to a standard lattice-Boltzmann scheme. Thus $p_{i}^{a d v}$ is computed from the probability density $f_{i}$ of fluid particles with the same discrete velocity $\mathbf{c}_{i}$ and the local density $\rho_{f}$ of fluid particles:

$$
p_{i}^{a d v}(\mathbf{r}, t)=\frac{f_{i}(\mathbf{r}, t)}{\rho_{f}(\mathbf{r}, t)}-w_{i}
$$

The terms between braces in Eq. (4) are specific to the tracer and include both the drift velocity $D \beta q \mathbf{E}$ under an applied electric field and the effect of conservative forces. These conservative forces are accounted for via the second term between braces. The last term in eq. (4) ensures detailed balance at steady state. This physical constraint is required to guarantee a proper computation of the VACF and other correlation functions. Previous schemes which made use of a linearized coupling between densities and electric fields, valid in the limit of small potential gradients $\left(\beta\left|\nabla V \cdot \mathbf{c}_{i} \Delta t\right| \ll 1\right)$, will not recover generally the appropriate VACF. Note that the new expression for $p_{i}$ also contains the effect of diffusion that arised from a constant term in the original algorithm, because the expansion of 
the Fermi function in powers of $\beta \nabla V \cdot \mathbf{c}_{i} \Delta t$ contains a constant $(1 / 2)$ term. The dimensionless parameter $\lambda$ determines the diffusion coefficient and hence the mobility of the tracer. Indeed, a Taylor expansion shows that Eqs. (3) and (4) propagate $P(\mathbf{r}, t)$ according to (1) with a diffusion coefficient $D=\lambda c_{s}^{2} \Delta t / 4$ with $c_{s}$ the speed of sound in the fluid, as was shown in Ref. [8] in the corresponding small gradient limit.

The probability density of the fluid required in (5) can be evolved using any lattice-Boltzmann scheme, such as the celebrated Bhatnagar-Gross-Krook collision operator [11]: $C\left[f_{i}\right]=f_{i}-\left(f_{i}-f_{i}^{e q}\right) / \tau$, with $f_{i}^{e q}$ the equilibrium probability density (which corresponds to a discretized, low-velocity expansion, of a Maxwellian), and $\tau$ the relaxation time. A particularly simple and illustrative example corresponds to the choice $\tau=1$, for which the fluid kinematic viscosity $c_{s}^{2} \Delta t / 2$. In this case $C\left[f_{i}\right]=f_{i}^{e q}$, i.e. to a first order expansion in the velocities [5]: $p_{i}^{a d v}(\mathbf{r}, t) \approx w_{i}(\mathbf{u}+\mathbf{a} \Delta t) \cdot \frac{\mathbf{c}_{\mathbf{i}}}{c_{s}^{2}}$, where $\mathbf{u}$ and $\mathbf{a}$ correspond to the local fluid velocity and accelerations, respectively. In the absence of coupling to a fluid, the first term in Eq. (4) vanishes and the tracer dynamics reduces to diffusion in the presence of electric fields. Let us conclude this discussion of the transition probabilities by a remark on the fluid-solid boundaries. Since the corresponding probability $p_{i}(\mathbf{r}, t)$ is zero, velocities leading to solid nodes do not contribute either to transport or to the average tracer velocity $\mathbf{u}^{*}(\mathbf{r}, t)=\sum_{i} p_{i}(\mathbf{r}, t) \mathbf{c}_{i}$, which to first order in $\beta\left\|\nabla V \cdot \mathbf{c}_{i} \Delta t\right\|$ reduces to $\mathbf{u}^{*}=\mathbf{u}+D \beta q \mathbf{E}-D \beta \nabla V$.

As has been emphasized in the introduction, we are not interested in using the moment propagation algorithm only to simulate a convection-diffusion equation for charged tracers, but also to compute their VACF. To this end, we take advantage of the fact that the tracer velocities are restricted to the finite set of allowed displacements, $\left\{\mathbf{c}_{i}\right\}$. Accordingly, the autocorrelation function of the $\gamma \in\{x, y, z\}$ component of the velocity can be expressed as a discrete sum over velocities at times 0 and $t$

$$
\begin{aligned}
Z_{\gamma}(t) & =\left\langle v_{\gamma}^{0} v_{\gamma}^{t}\right\rangle=\sum_{\mathbf{r}^{0}, c_{\gamma}^{0}} \pi\left(\mathbf{r}^{0}, c_{\gamma}^{0}\right) \pi\left(c_{\gamma}^{t} \mid \mathbf{r}^{0}, c_{\gamma}^{0}\right) c_{\gamma}^{t} c_{\gamma}^{0} \\
& =\sum_{\mathbf{r}^{0}, c_{\gamma}^{0}} \pi\left(\mathbf{r}^{0}, c_{\gamma}^{0}\right) c_{\gamma}^{0} \sum_{\mathbf{r}^{t}} \pi\left(\mathbf{r}^{t}, c_{\gamma}^{t} \mid \mathbf{r}^{0}, c_{\gamma}^{0}\right) c_{\gamma}^{t},
\end{aligned}
$$

where $\pi\left(\mathbf{r}^{0}, c_{\gamma}^{0}\right)$ gives the probability of being at node $\mathbf{r}^{0}$ with velocity $c_{\gamma}^{0}$ while $\pi\left(c_{\gamma}^{t} \mid \mathbf{r}^{0}, c_{\gamma}^{0}\right)$ stands for the corresponding conditional probability. The previous formulation allows us to quantify the probability of all possible paths that a tracer follows to arrive at node $\mathbf{r}$ and time $t$ with velocity $\mathbf{c}_{i}$, in a way similar to path integral methods. Taking the post-collisional velocity of a given tracer distributed according to probabilities $p_{i}(\mathbf{r}, t)$ independently of the pre-collisional velocity of that particle, we can replace $\pi\left(\mathbf{r}^{t}, c_{\gamma}^{t} \mid \mathbf{r}^{0}, c_{\gamma}^{0}\right) c_{\gamma}^{t}$ by the post-collisional average $\pi\left(\mathbf{r}^{t} \mid \mathbf{r}^{0}, c_{\gamma}^{0}\right) \sum_{i} p_{i}(\mathbf{r}, t) c_{i \gamma}=\pi\left(\mathbf{r}^{t} \mid \mathbf{r}^{0}, c_{\gamma}^{0}\right) u_{\gamma}^{*}(\mathbf{r}, t)$ to rewrite Eq. (6) as:

$$
\begin{aligned}
Z_{\gamma}(t) & =\sum_{\mathbf{r}^{t}}\left[\sum_{\mathbf{r}^{0}, c_{\gamma}^{0}} \pi\left(\mathbf{r}^{0}, c_{\gamma}^{0}\right) c_{\gamma}^{0} \pi\left(\mathbf{r}^{t} \mid \mathbf{r}^{0}, c_{\gamma}^{0}\right)\right] u_{\gamma}^{*}(\mathbf{r}, t) \\
& =\sum_{\mathbf{r}} P(\mathbf{r}, t ; \gamma) u_{\gamma}^{*}(\mathbf{r}, t)
\end{aligned}
$$

where we have introduced the probability to arrive at node $\mathbf{r}$ at time $t$ weighted by the $\gamma$ component of the initial velocity of the tracers $P(\mathbf{r}, t ; \gamma)$. Since these quantities (one per direction) are linear combinations of $\pi\left(\mathbf{r}^{t} \mid \mathbf{r}^{0}, c_{\gamma}^{0}\right)$, which is a solution of the linear advection-diffusion equation, it also evolves according to (1). This fact is exploited by the moment propagation method, which consists in propagating this weighted average by Eqs. (3) and (4) after initializing at time 1 as $P(\mathbf{r}, 1 ; \gamma)=\sum_{i} \frac{e^{-\beta V\left(\mathbf{r}-\mathbf{c}_{\mathbf{i}} \Delta t\right)}}{Q} p_{i}(\mathbf{r}-$ $\left.\mathbf{c}_{\mathbf{i}} \Delta t, 0\right) c_{i \gamma}$, with $Q=\sum_{\mathbf{r}} e^{-\beta V(\mathbf{r})}$. The average over all initial conditions in (6) is thus performed at once while propagating $P(\mathbf{r}, t ; \gamma)$. For the initial value of the VACF we simply have: $Z_{\gamma}(0)=\sum_{\mathbf{r}, i} \frac{e^{-\beta V(\mathbf{r})}}{Q} p_{i}(\mathbf{r}, 0) c_{i \gamma} c_{i \gamma}$, consistent with the need to start from an equilibrium tracer configuration.

In order to validate this new scheme, we consider the dispersion of charged tracers by electro-osmotic flow in a charged slit without added salt. Two plates of negative charge density $\sigma e$ with $e$ the elementary charge and $\sigma<0$ are located at $x= \pm L / 2$, and the monovalent counterions are dissolved in a solvent of viscosity $\eta$. The counterion concentration is (see e.g. [8]) $c_{+}(x)=\frac{\alpha^{2}}{2 \pi l_{B}} \frac{1}{\cos ^{2} \alpha x}$ where $\frac{\alpha L}{2} \tan \frac{\alpha L}{2}=\pi \sigma L l_{B}$, with $l_{B}=e^{2} / 4 \pi \epsilon_{0} \epsilon_{r} k_{B} T$ the Bjerrum length, while $\alpha^{-1}$ stands for the analog of the Debye screening length and characterizes the distorsion of the counterion profile. For small charge densities, $\alpha L \sim \sqrt{4 \pi \sigma L l_{B}}$, whereas at large charge densities $\alpha L \rightarrow \pi$. In presence of an applied electric field $E_{y} \mathbf{e}_{\mathbf{y}}$ an electro-osmotic flow profile arises

$$
u_{y}(x)=\frac{e E_{y}}{2 \pi \eta l_{B}} \ln \frac{\cos \alpha x}{\cos \alpha L / 2} \equiv u_{\mathrm{ref}} h(x)
$$

with characteristic velocity $u_{\text {ref }}=e E_{y} / 2 \pi \eta l_{B}$.

If we now consider the transport of tracers of charge $q=z e$ under the influence of both the electric field and the induced electro-osmotic flow, the average velocity is given by

$$
\bar{v}_{y, q}=\beta D q E_{y}+u_{\mathrm{ref}} \int_{-1 / 2}^{1 / 2} \mathrm{~d} \xi \mathcal{B}_{q}(\xi) h(\xi),
$$

where $\xi=x / L$ and $\mathcal{B}_{q}(x)$ is the normalized Boltzmann weight for a charge $q$ in the electrostatic potential: $\psi(x)=$ $\psi(L / 2)+\ln \left[\cos ^{2}(\alpha x) / \cos ^{2}(\alpha L / 2)\right]$. The dispersion of tracers around this average is characterized by a dispersion coefficient varying at sufficiently low Péclet number $P e=u_{\mathrm{ref}} L / D$ as

$$
\frac{D_{y}}{D}=1+f(\alpha L, q) \times P e^{2} .
$$


This expression comes from the fact that there is no linear contribution in $P e$ and defines the dispersion factor $f$ as the prefactor of the quadratic contribution, which depends on both the surface charge density and the tracer charge [12]. Since the dispersion tensor (2) is symmetric in this case, in the following we will consider only its diagonal components, and use the simplified notation $D_{\gamma} \equiv D_{\gamma \gamma}$. In the $x$ and $z$ directions the average velocity is zero. Thus the corresponding components reduce to the same components of the diffusion tensor. If the tracers can explore the whole slit section, the dispersion factor can be expressed as [12]

$$
\begin{aligned}
& f(\alpha L, q)=-\int_{-1 / 2}^{1 / 2} \mathrm{~d} \xi \mathcal{B}_{q}(\xi) g_{q}(\xi) \times \\
& \int_{0}^{\xi} \mathrm{d} \xi^{\prime} \frac{1}{\mathcal{B}_{q}\left(\xi^{\prime}\right)} \int_{0}^{\xi^{\prime}} \mathrm{d} \xi^{\prime \prime} \mathcal{B}_{q}\left(\xi^{\prime \prime}\right) g_{q}\left(\xi^{\prime \prime}\right)
\end{aligned}
$$

where the deviation from the average velocity $g_{q}(\xi)=$ $\left[u_{y}(\xi)+\beta D q E_{y}-\bar{v}_{y, q}\right] / u_{\text {ref }}$ depends on both the charge $q$ of the tracer and $\alpha L$ via the local velocity $u_{y}$.

Simulations are performed using the D3Q19 lattice for which $c_{s}^{2}=1 / 3(\Delta x / \Delta t)^{2}[5]$, on $N_{x} \times N_{y} \times N_{z}=62 \times 5 \times 5$ lattice points with a layer of solid nodes at $n_{x}=1$ and 62 . Bounce-back rules between fluid and solid nodes ensure the no-slip condition at the solid surface, and correspond to a distance between walls of $L=\left(N_{x}-2\right) \Delta x=60 \Delta x$. Periodic boundary conditions in $y$ and $z$ directions are used. To evolve the fluid probability density appearing in (5) we have used the BGK collision operator described above with a relaxation time $\tau=1$. This corresponds for the D3Q19 lattice to a kinematic viscosity of the solvent $\nu=1 / 6\left(\Delta x^{2} / \Delta t\right)$. Also, $\lambda$ is chosen to give a diffusion coefficient $D=0.05\left(\Delta x^{2} / \Delta t\right)$, such that the Schmidt number $S c=\nu / D$ is large, as expected for small ions in water. We choose a lattice spacing of $\Delta x=2.5 l_{B}$ to ensure sufficient sampling of the ionic concentrations in the regions where it varies most rapidly, i.e. near the surface over a distance $\alpha^{-1}$ which is in our case always large compared to $l_{B}$. For water at room temperature the Bjerrum length is $l_{B} \sim 7 \AA$ and it corresponds to a distance between walls of $L=60 \Delta x \sim 100 \mathrm{~nm}$. The electric field is applied along $y$, with $\beta e E_{y} \Delta x=0.0$ to 0.2 (only to 0.1 for $\alpha L=2.90$ and 3.02 , to ensure numerical stability). The potential $\psi$ is computed from the charge density with the Successive Over Relaxation method [13].

The counterion and steady-state flow profiles are first determined using the link-flux method [9]. The latter are reported in Fig. 1 for fixed applied electric field $\beta e E_{y} L=3$ as a function of $\alpha L$. Except for the highest charge density $(\alpha L \sim 3.02)$, the choice of simulation parameters allows to accurately reproduce the continuum results. The lack of accuracy for large $\alpha L$ values can be cured by increasing the number of lattice points and scaling the charge density accordingly.

The diagonal components of the VACF tensor $Z_{\gamma}(t)$ $(\gamma \in\{x, y, z\})$ for tracers of valence $-1,0$ and +1 are com-

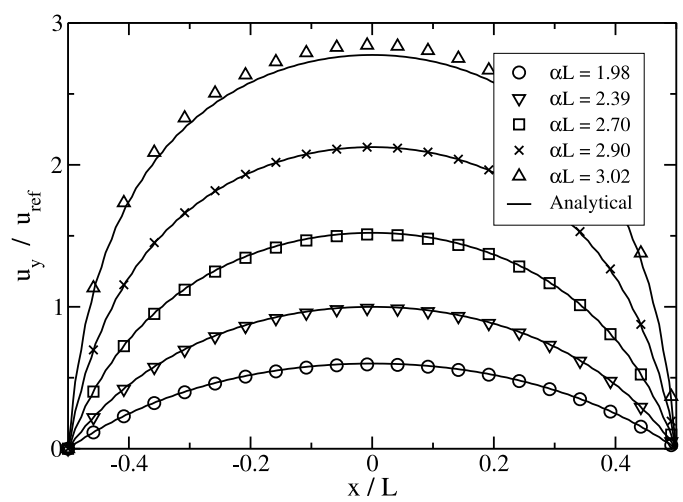

Fig. 1: Steady-state flow profile across the slit for an applied electric field $\beta e E_{y} L=3$ as a function of $\alpha L$. The results are normalized by $u_{\mathrm{ref}}=e E_{y} / 2 \pi \eta l_{B}$. Symbols are simulation results using the link flux method. The drawn curves have been computed on the basis of Eq. (8).

puted using the moment propagation algorithm with the steady-state velocity $\mathbf{u}$ and potential $V=q \psi$ for the scattering probabilities in (4) and (5). For the $z$ component of the VACF, one finds numerically that $Z_{z}(0)=2 D / \Delta t$ and $Z_{z}(t>0)=0$, i.e. the discrete version of the expected result $D \delta(t)$ for free Brownian motion. The diffusion coefficient in this direction $D_{z}=\int_{0}^{\infty} \mathrm{d} t Z_{z}(t)$ is thus equal to the molecular diffusion coefficient $D$, independently of $\alpha L$ and of the tracer charge. In the direction normal to the surface we also have $Z_{x}(0)=2 D / \Delta t$, but at long time a negative tail always leads to $\int_{0}^{\infty} \mathrm{d} t Z_{x}(t)=0$, because of the confinement by the surfaces.

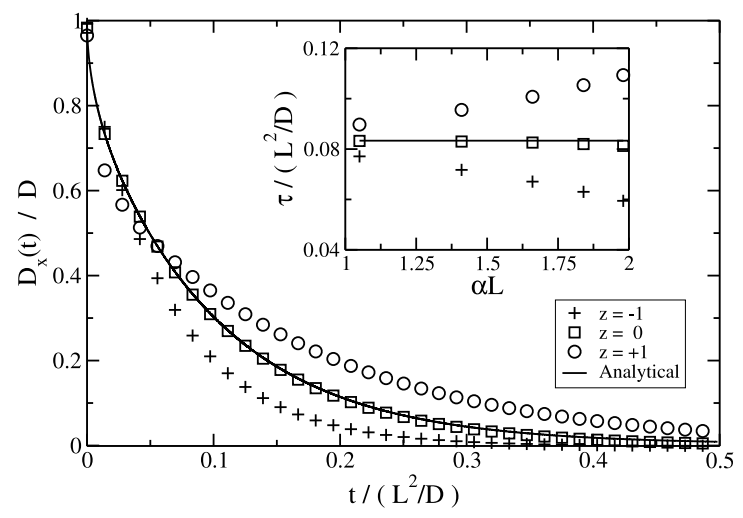

Fig. 2: Time-dependent diffusion coefficient in the direction normal to the surfaces $D_{x}(t)=\int_{0}^{t} \mathrm{~d} t^{\prime} Z_{x}\left(t^{\prime}\right)$ for anions $(+)$, neutral $(\square)$ and cations (o) for $\alpha L=1.98$. The line is the analytical result for neutral tracers. The inset shows the characteristic decay time $\tau=\int_{0}^{\infty} \mathrm{d} t D_{x}(t) / D$ as a function of $\alpha L$.

The time-dependence of $Z_{x}(t)$ does however vary with $\alpha L$ and tracer charge. This is more conveniently ana- 
lyzed in terms of the time-dependent diffusion coefficient $D_{x}(t)=\int_{0}^{t} \mathrm{~d} t^{\prime} Z_{x}\left(t^{\prime}\right)$, which can be experimentally measured with NMR using pulsed field gradients and often applied to quantify the surface to volume ratio of porous media [14]. Simulation results for $\alpha L=1.98$ are reported in Fig. 2 together with the analytical solution for neutral tracers: $D_{x}(t) / D=\frac{8}{\pi^{2}} \sum_{n=0}^{\infty} \frac{1}{(2 n+1)^{2}} \exp \left[-(2 n+1)^{2} \frac{\pi^{2} D t}{L^{2}}\right]$. $D_{x}(t)$ decays faster for anions than for neutral tracers, because the repulsion from the negatively charged walls confines them to a smaller region. This results in a faster memory loss of their initial velocity. After a fast decay, $D_{x}(t)$ for cations exhibits a long time tail: The initial part corresponds to confinement in the vicinity of one surface and the longer tail to the escape from one surface to the other.

These findings have implication for the time needed to consider that the motion can be averaged in the direction normal to the surfaces, i.e. to the flow, which is the assumption underlying Eq. (10b). This time can be estimated as $\tau=\int_{0}^{\infty} \mathrm{d} t D_{x}(t) / D$ and is reported in the inset of Fig. 2 as a function of $\alpha L$. It is seen to be constant for neutral species (integration of the analytical expression given in the previous paragraph gives $L^{2} / 12 D$ ), shorter and decreasing for anions, while longer and increasing for cations. It is worth emphasizing that although the timedependent diffusion coefficient of neutral tracers has already been computed in complex porous media [15], no such determination has been made in the case of charged species. The present method offers a way to address this issue.

The average velocity of each tracer can be obtained from $\bar{v}_{y, q}=\sqrt{Z_{y, q}(\infty)}$. It is reported as a function of the applied electric field in Fig. 3, which illustrates the quantitative agreement of simulation results with Eq. (9). For our choice of simulation parameters (corresponding to small ions in water and a distance between surfaces of $\sim 100 \mathrm{~nm}$ ), the electro-osmotic flow is larger than the direct electrostatic contribution $\beta D q E$ to the average velocity. The average velocity is larger for co-ions (here anions), which are mainly located near the center of the slit where the flow velocity is larger, than for neutral tracers or counterions (here cations), which are concentrated near the surfaces where the flow vanishes. Increasing the charge density increases both the average velocities and the difference between tracers of different charge. This last effect arises because the velocity profiles are more inhomogeneous for larger charge densities, as suggested by Eq. (8).

The dispersion coefficient along the flow is computed from the VACF via (2) as $D_{y, q}=$ $\int_{0}^{\infty} \mathrm{d} t\left[Z_{y, q}(t)-Z_{y, q}(\infty)\right]$, for each tracer charge $q$. Dispersion is caused by the crossing of streamlines in the direction normal to the surfaces and is thus affected by the electric potential gradient in this direction. The agreement of the moment propagation results in Fig. 4 with the continuous prediction of Eq. (10) is seen to be

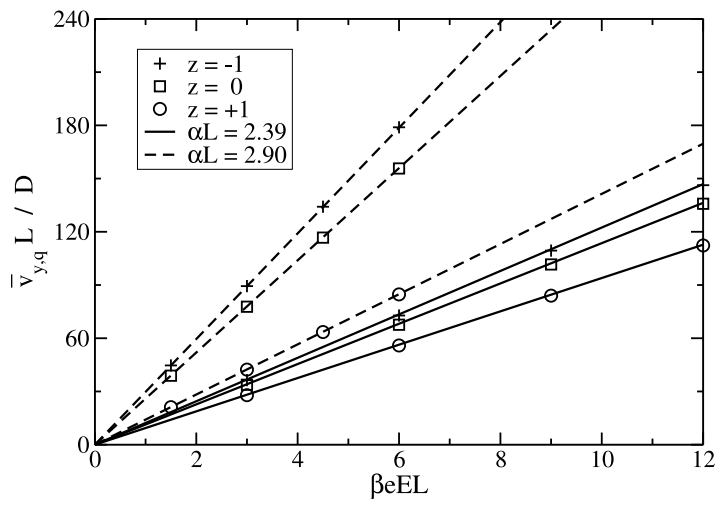

Fig. 3: Average velocity under an applied electric field, for negatively charged surfaces. Symbols are the simulation results, lines that of Eq. (9). The electro-osmotic flow is larger than the direct electrostatic contribution $\beta D q E$ : Anions (+), mainly located in the center of the slit where the flow is faster, have a larger average velocity than neutral tracers ( $\square$ ) and cations (o), the latter being concentrated near the surfaces, where the flow vanishes. Increasing the charge density from $\alpha L=2.39$ (solid line) to 2.90 (dashed line) incresases both the average velocities and the difference between tracers of different charge.

excellent. Anions are less dispersed than neutral tracers and cations because they are concentrated in the center of the slit, where the flow is less inhomogeneous. Increasing the charge density has a larger effect on counterions (cations) and neutral tracers than on co-ions (anions).

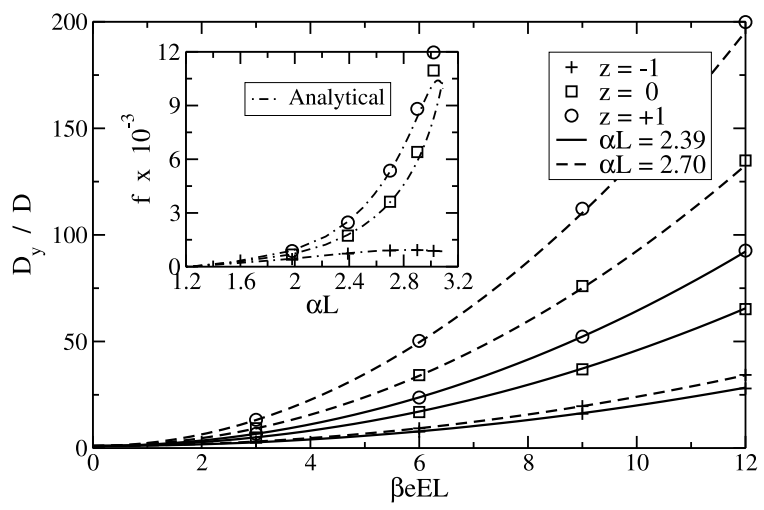

Fig. 4: Dispersion coefficient from moment propagation (symbols) and Eq. (10) (lines). Anions $(+)$ are less dispersed than neutral $(\square)$ and cations (o), because they are concentrated in the center of the slit, where the flow is more homogeneous. Increasing the charge density incresases both the dispersion coefficient and the difference between tracers of different charge. Inset: dispersion factor $f$ obtained by fitting $D_{y} / D$ using Eq. (10a) for different values of $\alpha L$; lines are results of Eq. (10b).

From the dispersion coefficient we finally compute the 
dispersion factors defined by Eq. (10a) and compare them to the approximate analytical solution (10b). Results are reported in the inset of Fig. 4. Except for the highest charge density (corresponding to $\alpha L \sim 3.02$ ) the agreement is again quantitative. The discrepancy for the extreme case is not due to the breakdown of the approximations leading to (10b), but to an effect of the lattice already visible on the flow profile (Fig. 1). Increasing the number of lattice points while maintaining $\alpha L$ constant leads to better agreement, but requires longer simulation times.

We have proposed a new method to study efficiently the VACF of charged tracers, based on the moment propagation method. We have used previous proposals which make use of the lattice-Boltzmann approach to study the dynamics of electrolytes and have proposed a transition probability for tracers which ensures detailed balance at steady-state. This has made it possible to compute the statistical properties of tracer trajectories following the underlying dynamics of the charged fluid. Such an approach is based on the transition probabilities between neighboring lattice sites defined by Eqs. (4) and (5). The algorithm has been validated by studying the dispersion in an electro-osmotic flow between charged walls without added salt. Previous studies have only focused on effective treatments of the electrokinetic couplings present in porous media [16]. Therefore, the proposed generalization significantly widens the application range of the moment propagation method; it has wide implications, since charged porous media are ubiquitous. Moreover, the transition probabilities introduced constitute the basis for the study of tracer statistics in non-ideal fluids. The method benefits from all the advantages offered by its latticeBoltzmann nature, in particular for the handling of various hydrodynamic conditions and the ease of parallelization. Furthermore, moment propagation can be applied to compute other correlation functions. For example, it has been used to compute the dynamic structure factor of uncharged tracers [6]. The extension to the charged tracer case from the results of the present paper is straightforward. Future work will be devoted to the study of diffusion and dispersion of charged tracers in more complex porous media. The computation of the time-dependent diffusion coefficient made possible by this algorithm will then be of particular interest, since it reflects the different porosities probed by tracers of different charge and it can be measured by NMR.

\section{Acknowledgments}

The authors would like to thank Fabrizio Capuani for providing his Lattice Electrokinetics code, and Daniel Coelho for useful discussions. The work of the FOM Institute is part of the research program of FOM and is made possible by financial support from the Netherlands organization for Scientific Research (NWO). B.R. acknowledges financial support from the Agence Nationale pour la Gestion des Déchets Radioactifs (ANDRA, France). I.P. acknowledges financial support from CAICYT (FIS2005-01299) and from "Distinció de la Generalitat de Catalunya".

\section{REFERENCES}

[1] Lowe C. and Frenkel D., Phys. Rev Lett. , 77 (1996) 4552.

[2] Capunin F., Frenkel D. and Lowe C., Phys. Rev E, 67 (2003) 056306.

[3] Van der Hoef M. and Frenkel D., Phys. Rev. A , $4 \mathbf{1}$ (1990) 4277.

[4] Merks R., Hoekstra A. and Sloot P., J. Comput. Phys., 183 (2002) 563.

[5] Succi S., The Lattice Boltzmann Equation for Fluid Dynamics and Beyond (Oxford University Press, Oxford) 2001.

[6] Lowe C. P., Frenkel D. and Van der Hoef M., $J$. Stat. Phys. , 87 (1997) 1129.

[7] Rasin I., Succi S. and Miller W., J. Comput. Phys., 206 (2005) 453.

[8] Warren P., Int. J. Mod. Phys. C, 8 (1997) 889.

[9] Capuani F., Pagonabarraga I. and Frenkel D., $J$. Chem. Phys. , 121 (2004) 973.

[10] Benzi R., Succi S. and Vergassola M., Phys. Rep., 222 (1992) 145.

[11] Bhatnagar P., Gross E. and Krook M., Phys. Rev., 94 (1954) 511.

[12] De Leebeeck A. and Sinton D., Electrophoresis , 27 (2006) 4999.

[13] Press W. H., Teukolsky S. A., Vetterling W. T. and Flannery B. P., Numerical Recipes in C: The Art of Scientific Computing 2nd Edition (Cambridge University Press, Cambridge) 1993.

[14] Sen P., Conc. Magn. Reson. Part A, 23A (2004) 1.

[15] Valfouskaya A., Adler P., Thovert J.-F. and Fleury M., J. App. Phys., 97 (2005) 083510.

[16] Hlushkou D., Khirevich S., Apanasovich V., SeidelMorgenstern A. and Tallarek U., Anal. Chem., 90 (2007) 113. 